Article

\title{
On Some Formulas for the $k$-Analogue of Appell Functions and Generating Relations via $k$-Fractional Derivative
}

\author{
Övgü Gürel Yılmaz ${ }^{1}\left(\mathbb{D}\right.$, Rabia Aktaş ${ }^{2, * \mathbb{C}}$ and Fatma Taşdelen ${ }^{2}$ (D) \\ 1 Department of Mathematics, Recep Tayyip Erdogan University, 53100 Rize, Turkey; \\ ovgu.gurelyilmaz@erdogan.edu.tr \\ 2 Department of Mathematics, Faculty of Science, Ankara University, Tandoğan, 06100 Ankara, Turkey; \\ tasdelen@science.ankara.edu.tr \\ * Correspondence: raktas@science.ankara.edu.tr
}

Received: 21 August 2020; Accepted: 22 September 2020; Published: 24 September 2020

check for updates

\begin{abstract}
Our present investigation is mainly based on the $k$-hypergeometric functions which are constructed by making use of the Pochhammer $k$-symbol in Diaz et al. 2007, which are one of the vital generalizations of hypergeometric functions. In this study, we focus on the $k$-analogue of $F_{1}$ Appell function introduced by Mubeen et al. 2015 and the $k$-generalizations of $F_{2}$ and $F_{3}$ Appell functions indicated in Kiymaz et al. 2017. We present some important transformation formulas and some reduction formulas which show close relation not only with $k$-Appell functions but also with $k$-hypergeometric functions. Employing the theory of Riemann-Liouville $k$-fractional derivative from Rahman et al. 2020, and using the relations which we consider in this paper, we acquire linear and bilinear generating relations for $k$-analogue of hypergeometric functions and Appell functions.
\end{abstract}

Keywords: $k$-gamma function; $k$-beta function; Pochhammer symbol; hypergeometric function; Appell functions; integral representation; reduction and transformation formula; fractional derivative; generating function

\section{Introduction}

Special functions, with their diverse sub-branches, provide a very wide field of study that appears not only in various fields of mathematics, but also in the solutions of important problems in many disciplines of science such as physics, chemistry, and biology. This subject is powerful enough to make sense of uncertain questions especially in physical problems, so it encourages many people for notable improvements on this matter. As in other sciences, remarkable problems are still discussed in many disciplines, and more general results are attempted to be obtained.

Generalized hypergeometric functions, which are some of these studies in special functions $[1,2]$, are defined by

$$
{ }_{p} F_{q}\left[\begin{array}{c}
\alpha_{1}, \alpha_{2}, \ldots, \alpha_{p} \\
\beta_{1}, \beta_{2}, \ldots, \beta_{q}
\end{array} ; x\right]=\sum_{n=0}^{\infty} \frac{\left(\alpha_{1}\right)_{n}\left(\alpha_{2}\right)_{n} \ldots\left(\alpha_{p}\right)_{n}}{\left(\beta_{1}\right)_{n}\left(\beta_{2}\right)_{n} \ldots\left(\beta_{q}\right)_{n}} \frac{x^{n}}{n !},
$$

where $\alpha_{1}, \alpha_{2}, \ldots, \alpha_{p}, \beta_{1}, \beta_{2}, \ldots, \beta_{q}, x \in \mathbb{C}$ and $\beta_{1}, \beta_{2}, \ldots, \beta_{q}$ are neither zero nor negative integers.

Here, $(\lambda)_{n}$ is the Pochhammer symbol defined by

$$
(\lambda)_{n}=\left\{\begin{array}{cc}
\lambda(\lambda+1) \ldots(\lambda+n-1) ; & n \geq 1 \\
1 \quad ; n=0, \lambda \neq 0 .
\end{array}\right.
$$


For the special case that corresponds to $p=2$ and $q=1$ in (1), we can obtain ${ }_{2} F_{1}$ Gauss hypergeometric function [1,2],

$$
{ }_{2} F_{1}\left[\begin{array}{c}
\alpha, \beta \\
\gamma
\end{array} ; x\right]=\sum_{n=0}^{\infty} \frac{(\alpha)_{n}(\beta)_{n}}{(\gamma)_{n}} \frac{x^{n}}{n !}, \quad|x|<1,
$$

where $\alpha, \beta, \gamma, x \in \mathbb{C}$ and $\gamma$ is neither zero nor a negative integer.

Many elementary functions can be expressed in terms of hypergeometric functions. Moreover, non-elementary functions that occur in physics and mathematics have a representation with hypergeometric series. Therefore, generalizing hypergeometric functions have many applications in mathematics and the other disciplines. For instance, in quantum field theory, hypergeometric functions appear in the calculation of the Feynmann integrals, and the analytic results can be expressed in terms of these functions [3,4]. In engineering, analytic forms of the fractional order derivatives of sinusoidal functions are represented with hypergeometric functions [5]. In biochemistry, for the analysis of a simple gene expression model, a hypergeometric probability distribution is considered [6], and they also appear in the connection and linearization problems in mathematics [7-9]. Generalization of hypergeometric function can be made by increasing the number of parameters in the hypergeometric function or by increasing the number of variables. Appell, based on the idea that the number of variables can be increased, has defined Appell hypergeometric functions obtained by multiplying two hypergeometric functions. These are the four elemanter functions defined in [1,2]

$$
\begin{aligned}
F_{1}\left(\alpha, \beta, \beta^{\prime} ; \gamma ; x, y\right) & =\sum_{m, n=0}^{\infty} \frac{(\alpha)_{m+n}(\beta)_{m}\left(\beta^{\prime}\right)_{n}}{(\gamma)_{m+n}} \frac{x^{m}}{m !} \frac{y^{n}}{n !}, \\
F_{2}\left(\alpha, \beta, \beta^{\prime} ; \gamma, \gamma^{\prime} ; x, y\right) & =\sum_{m, n=0}^{\infty} \frac{(\alpha)_{m+n}(\beta)_{m}\left(\beta^{\prime}\right)_{n}}{(\gamma)_{m}\left(\gamma^{\prime}\right)_{n}} \frac{x^{m}}{m !} \frac{y^{n}}{n !}, \\
F_{3}\left(\alpha, \alpha^{\prime}, \beta, \beta^{\prime} ; \gamma ; x, y\right) & =\sum_{m, n=0}^{\infty} \frac{(\alpha)_{m}\left(\alpha^{\prime}\right)_{n}(\beta)_{m}\left(\beta^{\prime}\right)_{n}}{(\gamma)_{m+n}} \frac{x^{m}}{m !} \frac{y^{n}}{n !}, \\
F_{4}\left(\alpha, \beta ; \gamma, \gamma^{\prime} ; x, y\right) & =\sum_{m, n=0}^{\infty} \frac{(\alpha)_{m+n}(\beta)_{m+n}}{(\gamma)_{m}\left(\gamma^{\prime}\right)_{n}} \frac{x^{m}}{m !} \frac{y^{n}}{n !},
\end{aligned}
$$

where $|x|<1,|y|<1 ;|x|+|y|<1 ;|x|<1,|y|<1 ; \sqrt{|x|}+\sqrt{|y|}<1$, respectively. Appell functions can be found in the study of autoionization of atoms [10], separability of Hamilton-Jacobi equations in classical mechanics [11], representation of Feynmann integral in quantum field theory [3,4], and expression of Nordsieck integral in atomic collisions physics [12].

Another generalization of hypergeometric functions is the hypergeometric $k$-function, defined by the Pochhammer $k$-symbol studied by Diaz et al. [13]. This paper includes the $k$-analogue of the Pochhammer symbol and hypergeometric function, as well as the $k$-generalization of gamma, beta, and zeta functions with their integral representations and some identities provided by classical ones. It should be noted that, taking $k=1$ in these generalizations, the $k$-extensions of the functions reduce to the classical ones.

Let $k \in \mathbb{R}^{+}$and $n \in \mathbb{N}^{+}$. Hypergeometric $k$-function is defined in [13] as

$$
{ }_{2} F_{1, k}\left[\begin{array}{c}
\alpha, \beta \\
\gamma
\end{array} ; x\right]:={ }_{2} F_{1, k}\left[\begin{array}{c}
(\alpha, k),(\beta, k) \\
(\gamma, k)
\end{array} ; x\right]=\sum_{n=0}^{\infty} \frac{(\alpha)_{n, k}(\beta)_{n, k}}{(\gamma)_{n, k}} \frac{x^{n}}{n !},
$$

where $\alpha, \beta, \gamma, x \in \mathbb{C}$ and $\gamma$ neither zero nor a negative integer and $(\lambda)_{n, k}$ is the Pochhammer $k$-symbol defined in [13] as

$$
(\lambda)_{n, k}=\left\{\begin{array}{cc}
\lambda(\lambda+k)(\lambda+2 k) \ldots(\lambda+(n-1) k) ; & n \geq 1, \\
1 \quad ; n=0, \lambda \neq 0 .
\end{array}\right.
$$


Based on this generalization, Kokologiannaki [14] obtained different inequalities and properties for the generalizations of Gamma, Beta, and Zeta functions. Some limits with the help of asymptotic properties of $k$-gamma and $k$-beta functions were discussed by Krasniqi [15]. Mubeen et al. [16] established integral representations of the $k$-confluent hypergeometric function and $k$-hypergeometric function and, in another paper [17], proved the $k$-analogue of the Kummer's first formulation using these integral representations. In [18], some families of multilinear and multilateral generating functions for the $k$-analogue of the hypergeometric functions were obtained. Studies on this subject are not limited to these papers; for details, see [19-22].

In [23], Mubeen adapted the $k$-generalization to the Riemann-Liouville fractional integral by using $k$-gamma function. In [24], $k$-Riemann-Liouville fractional derivative was studied and new properties were obtained with the help of Fourier and Laplace transforms. In [25], Rahman et al. applied the newly $k$-fractional derivative operator to $k$-analogue of hypergeometric and Appell functions and obtained new relations satisfied between them. Furthermore, $k$-fractional derivative operator was applied to the $k$-Mittag-Leffler function and the Wright function.

Our present investigation is motivated by the fact that generalizations of hypergeometric functions have considerable importance due to their applications in many disciplines from different perspectives. Therefore, our study is generally based on the $k$-extension of hypergeometric functions. The structure of the paper is organized as follows: In Section 2, we briefly give some definitions and preliminary results which are essential in the following sections as noted in $[13,23,26,27]$. In Section 3, we prove some main properties such as transformation formulas, and some reduction formulas which enable us to have relations for $k$-hypergeometric functions and $k$-Appell functions. In the last part of the paper, applying the theory of Riemann-Liouville $k$-fractional derivative [25] and using the relations which we consider in the previous sections, we gain linear and bilinear generating relations for $k$-analogue of hypergeometric functions and $k$-Appell functions.

\section{Some Definitions and Preliminary Results}

For the sake of completeness, it will be better to examine the preliminary section in three subsections by the reason of the number of theorems and definitions. In these subsections, we will present some definitions, properties, and results which we need in our investigation in further sections. We begin by introducing $k$-gamma, $k$-beta, and $k$-analogue of hypergeometric function and we continue definitions of $k$-generalized $F_{1}, F_{2}$ and $F_{3}$ which are the classical Appell functions. We conclude this section with recalling Riemann-Liouville fractional derivative, $k$-generalization of this fractional derivative, and some important theorems which will be required in our studies.

Through this paper, we denote by $\mathbb{C}, \mathbb{R}, \mathbb{R}^{+}, \mathbb{N}$ and $\mathbb{N}^{+}$the sets of complex numbers, real numbers, real and positive numbers, and positive integers with zero and positive integers, respectively.

\section{1. $k$-Generalizations of Gamma, Beta, and Hypergeometric Functions}

In this subsection, the definitions of $k$-gamma and $k$-beta functions are presented and some elemental relations provided by these functions are introduced by Diaz et al. [13] and Mubeen et al. [22]. Furthermore, we continue the definition of $k$-hypergeometric function and we present integral representation and some formulas satisfied from this generalization [16,17].

Definition 1. For $x \in \mathbb{C}$ and $k \in \mathbb{R}^{+}$, the integral representation of $k$-gamma function $\Gamma_{k}$ is defined by

$$
\Gamma_{k}(x)=\int_{0}^{\infty} t^{x-1} e^{-\frac{t^{k}}{k}} d t
$$

where $\Re(x)>0[13,22]$. 
Definition 2. For $x, y \in \mathbb{C}$ and $k \in \mathbb{R}^{+}$, the $k$-beta function $B_{k}$ is defined by

$$
B_{k}(x, y)=\frac{1}{k} \int_{0}^{1} t^{\frac{x}{k}-1}(1-t)^{\frac{y}{k}-1} d t
$$

where $\Re(x)>0$ and $\Re(y)>0$ [13].

Proposition 1. Let $k \in \mathbb{R}^{+}, a \in \mathbb{R}, n \in \mathbb{N}^{+}$. The $k$-gamma function $\Gamma_{k}$ and the $k$-beta function $B_{k}$ satisfy the following properties [13,22],

$$
\begin{aligned}
\Gamma_{k}(x+k) & =x \Gamma_{k}(x), \\
\Gamma_{k}(x) & =k^{\frac{x}{k}-1} \Gamma\left(\frac{x}{k}\right), \\
B_{k}(x, y) & =\frac{\Gamma_{k}(x) \Gamma_{k}(y)}{\Gamma_{k}(x+y)}, \\
B_{k}(x, y) & =\frac{1}{k} B\left(\frac{x}{k}, \frac{y}{k}\right) .
\end{aligned}
$$

Definition 3. Let $x \in \mathbb{C}, k \in \mathbb{R}^{+}$and $n \in \mathbb{N}^{+}$. Then, the Pochhammer $k$-symbol is defined in [13,22] by

$$
(x)_{n, k}=x(x+k)(x+2 k) \ldots(x+(n-1) k) .
$$

In particular, we denote $(x)_{0, k}:=1$.

Proposition 2. If $\alpha \in \mathbb{C}$ and $m, n \in \mathbb{N}^{+}$then for $k \in \mathbb{R}^{+}$, we have

$$
\begin{aligned}
(\alpha)_{n, k} & =\frac{\Gamma_{k}(\alpha+n k)}{\Gamma_{k}(\alpha)} \\
(\alpha)_{n, k} & =k^{n}\left(\frac{\alpha}{k}\right)_{n} \\
(\alpha)_{m+n, k} & =(\alpha)_{m, k}(\alpha+m k)_{n, k}
\end{aligned}
$$

where $(\alpha)_{n}$ and $(\alpha)_{n, k}$ denote the Pochhammer symbol and Pochhammer k-symbol, respectively [13,22].

Proposition 3. For any $\alpha \in \mathbb{C}$ and $k \in \mathbb{R}^{+}$, the following identity holds

$$
\sum_{n=0}^{\infty}(\alpha)_{n, k} \frac{x^{n}}{n !}=(1-k x)^{-\frac{\alpha}{k}}
$$

where $|x|<\frac{1}{k}[13,22]$.

Theorem 1. Assume that $x \in \mathbb{C}, k \in \mathbb{R}^{+}$and $\Re(\gamma)>\Re(\beta)>0$, then the integral representation of the $k$-hypergeometric function is defined in [16] as

$$
{ }_{2} F_{1, k}\left[\begin{array}{c}
\alpha, \beta \\
\gamma
\end{array} ; x\right]=\frac{\Gamma_{k}(\gamma)}{k \Gamma_{k}(\beta) \Gamma_{k}(\gamma-\beta)} \int_{0}^{1} t^{\frac{\beta}{k}-1}(1-t)^{\frac{\gamma-\beta}{k}-1}(1-k x t)^{-\frac{\alpha}{k}} d t
$$

For the following theorem, ${ }_{2} F_{1, k}\left[\begin{array}{c}(\alpha, 1),(\beta, k) ; x \\ (\gamma, k)\end{array}\right]$ is the expression of the following form [17],

$$
{ }_{2} F_{1, k}^{*}\left[\begin{array}{c}
\alpha, \beta \\
\gamma
\end{array} ; x\right]:={ }_{2} F_{1, k}\left[\begin{array}{c}
(\alpha, 1),(\beta, k) ; x \\
(\gamma, k)
\end{array}\right]=\sum_{n=0}^{\infty} \frac{(\alpha)_{n}(\beta)_{n, k}}{(\gamma)_{n, k}} \frac{x^{n}}{n !}
$$


Theorem 2. In [17], assume that $x \in \mathbb{C}, k \in \mathbb{R}^{+}$and $\operatorname{Re}(\gamma-\beta)>0$, then

$$
{ }_{2} F_{1, k}\left[\begin{array}{c}
(\alpha, 1),(\beta, k) \\
(\gamma, k)
\end{array} ; x\right]:=\frac{\Gamma_{k}(\gamma) \Gamma_{k}(\gamma-\beta-k \alpha)}{\Gamma_{k}(\gamma-\beta) \Gamma_{k}(\gamma-k \alpha)} .
$$

For the special case $\alpha=-n$,

$$
{ }_{2} F_{1, k}\left[\begin{array}{c}
(-n, 1),(\beta, k) \\
(\gamma, k)
\end{array} ; x\right]=\frac{(\gamma-\beta)_{n, k}}{(\gamma)_{n, k}}
$$

2.2. k-Generalizations of the Appell Functions $F_{1}, F_{2}$ and $F_{3}$

In 2015, $k$-generalization of $F_{1}$ Appell function was introduced and contiguous function relations and integral representation of this function were shown by using the fundamental relations of the Pochhammer $k$-symbol [26]. In 2017, $k$-analogues of the $F_{2}, F_{3}$, and $F_{4}$ were explored by Kiymaz et al. in [27] and also in that study, they provided the relations between $k$-analogues of Appell functions and the classical ones. Here, we remind the definitions of $k$-analogue of $F_{1}, F_{2}$ and $F_{3}$ which are the Appell functions, and integral representations which are satisfied by them $[26,27]$.

Definition 4. In [26], let $k \in \mathbb{R}^{+}, x, y \in \mathbb{C}, \alpha, \beta, \beta^{\prime}, \gamma \in \mathbb{C}$ and $n \in \mathbb{N}^{+}$. Then, the $F_{1, k}$ function with the parameters $\alpha, \beta, \beta^{\prime}, \gamma$ is given by

$$
F_{1, k}\left(\alpha, \beta, \beta^{\prime} ; \gamma ; x, y\right)=\sum_{m, n=0}^{\infty} \frac{(\alpha)_{m+n, k}(\beta)_{m, k}\left(\beta^{\prime}\right)_{n, k}}{(\gamma)_{m+n, k}} \frac{x^{m}}{m !} \frac{y^{n}}{n !}
$$

where $\gamma \neq 0,-1,-2, \ldots$ and $|x|<\frac{1}{k},|y|<\frac{1}{k}$.

Definition 5. In [27], let $k \in \mathbb{R}^{+}, x, y \in \mathbb{C}, \alpha, \beta, \beta^{\prime}, \gamma, \gamma^{\prime} \in \mathbb{C}$ and $m, n \in \mathbb{N}^{+}$. Then, the Appell $k$-functions are defined by

$$
\begin{aligned}
F_{2, k}\left(\alpha, \beta, \beta^{\prime} ; \gamma, \gamma^{\prime} ; x, y\right) & =\sum_{m, n=0}^{\infty} \frac{(\alpha)_{m+n, k}(\beta)_{m, k}\left(\beta^{\prime}\right)_{n, k}}{(\gamma)_{m, k}\left(\gamma^{\prime}\right)_{n, k}} \frac{x^{m}}{m !} \frac{y^{n}}{n !} \\
F_{3, k}\left(\alpha, \alpha^{\prime}, \beta, \beta^{\prime} ; \gamma ; x, y\right) & =\sum_{m, n=0}^{\infty} \frac{(\alpha)_{m, k}\left(\alpha^{\prime}\right)_{n, k}(\beta)_{m, k}\left(\beta^{\prime}\right)_{n, k}}{(\gamma)_{m+n, k}} \frac{x^{m}}{m !} \frac{y^{n}}{n !} \\
F_{4, k}\left(\alpha, \beta ; \gamma, \gamma^{\prime} ; x, y\right) & =\sum_{m, n=0}^{\infty} \frac{(\alpha)_{m+n, k}(\beta)_{m+n, k}}{(\gamma)_{m, k}\left(\gamma^{\prime}\right)_{n, k}} \frac{x^{m}}{m !} \frac{y^{n}}{n !}
\end{aligned}
$$

where $|x|+|y|<\frac{1}{k} ;|x|<\frac{1}{k},|y|<\frac{1}{k} ; \sqrt{|x|}+\sqrt{|y|}<\frac{1}{\sqrt{k}}$, respectively, and denominators are neither zero nor negative integers.

Theorem 3. In [26], assume that $k \in \mathbb{R}^{+}, x, y \in \mathbb{C}, \Re(\gamma)>\Re(\alpha)>0$, then the integral representation of the $k$-hypergeometric function is as follows:

$$
\begin{aligned}
& F_{1, k}\left(\alpha, \beta, \beta^{\prime} ; \gamma ; x, y\right) \\
= & \frac{\Gamma_{k}(\gamma)}{k \Gamma_{k}(\alpha) \Gamma_{k}(\gamma-\alpha)} \int_{0}^{1} t^{\frac{\alpha}{k}-1}(1-t)^{\frac{\gamma-\alpha}{k}-1}(1-k x t)^{-\frac{\beta}{k}}(1-k y t)^{-\frac{\beta^{\prime}}{k}} d t .
\end{aligned}
$$


Theorem 4. In [27], let $k \in \mathbb{R}^{+}$. Integral representations of $F_{2, k}$ and $F_{3, k}$ have the forms of

$$
\begin{aligned}
& F_{2, k}\left(\alpha, \beta, \beta^{\prime} ; \gamma, \gamma^{\prime} ; x, y\right)=\frac{1}{k^{2} B_{k}(\beta, \gamma-\beta) B_{k}\left(\beta^{\prime}, \gamma^{\prime}-\beta^{\prime}\right)} \\
& \times \int_{0}^{1} \int_{0}^{1} \frac{t \frac{\beta}{k}-1 s^{\frac{\beta^{\prime}}{k}-1}(1-t)^{\frac{\gamma-\beta}{k}-1}(1-s)^{\frac{\gamma^{\prime}-\beta^{\prime}}{k}-1}}{(1-k x t-k y s)^{\frac{\alpha}{k}}} d t d s, \\
& F_{3, k}\left(\alpha, \alpha^{\prime}, \beta, \beta^{\prime} ; \gamma ; x, y\right)=\frac{\Gamma_{k}(\gamma)}{k^{2} \Gamma_{k}(\beta) \Gamma_{k}\left(\beta^{\prime}\right) \Gamma_{k}\left(\gamma-\beta-\beta^{\prime}\right)} \\
& \times \iint_{D} \frac{t^{\frac{\beta}{k}-1} s^{\frac{\beta^{\prime}}{k}-1}(1-k x t)^{-\frac{\alpha}{k}}(1-k y s)^{-\frac{\alpha^{\prime}}{k}}}{(1-t-s)^{1-\frac{\gamma-\beta-\beta^{\prime}}{k}}} d t d s,
\end{aligned}
$$

where $\Re(\gamma)>\Re(\beta)>0, \Re\left(\gamma^{\prime}\right)>\Re\left(\beta^{\prime}\right)>0$ and $D=\{t \geq 0, s \geq 0, t+s \leq 1\}$.

\subsection{The Riemann-Liouville k-Fractional Derivative Operator}

Fractional calculus and its applications have been intensively investigated for a long time by many researchers in numerous disciplines and attention to this subject has grown tremendously. By making use of the concept of the fractional derivatives and integrals, various extensions of them have been introduced [28-31], and authors have gained different perspectives in many areas such as engineering, physics, economics, biology, statistics [32,33]. One of the generalizations of fractional derivatives is Riemann-Liouville $k$-fractional derivative operator studied in $[24,25,34]$. Here, we remind the definition of Riemann-Liouville fractional derivative and its $k$-generalization and also some theorems which will be used in the further section, are displayed.

Definition 6. In [2], the well-known Riemann-Liouville fractional derivative of order $\mu$ is described, for a function $f$, as follows:

$$
\mathcal{D}_{z}^{\mu}\{f(z)\}=\frac{1}{\Gamma(-\mu)} \int_{0}^{z} f(t)(z-t)^{-\mu-1} d t
$$

where $\Re(\mu)<0$.

In particular, for the case $m-1<\Re(\mu)<m$, where $m=1,2, \ldots$ (32) is written by

$$
\begin{aligned}
\mathcal{D}_{z}^{\mu}\{f(z)\} & =\frac{d^{m}}{d z^{m}} \mathcal{D}_{z}^{\mu-m}\{f(z)\} \\
& =\frac{d^{m}}{d z^{m}}\left\{\frac{1}{\Gamma(-\mu+m)} \int_{0}^{x} f(t)(z-t)^{-\mu+m-1} d t\right\} .
\end{aligned}
$$

Definition 7. In [25], the k-analogue of Riemann-Liouville fractional derivative of order $\mu$ is defined by

$$
{ }_{k} \mathcal{D}_{z}^{\mu}\{f(z)\}=\frac{1}{k \Gamma_{k}(-\mu)} \int_{0}^{z} f(t)(z-t)^{-\frac{\mu}{k}-1} d t,
$$

where $\Re(\mu)<0$ and $k \in \mathbb{R}^{+}$. 
In particular, for the case $m-1<\Re(\mu)<m$, where $m=1,2, \ldots$, (34) is written by

$$
\begin{aligned}
{ }_{k} \mathcal{D}_{z}^{\mu}\{f(z)\} & =\frac{d^{m}}{d z^{m}}{ }_{k} \mathcal{D}_{z}^{\mu-m k}\{f(z)\} \\
& =\frac{d^{m}}{d z^{m}}\left\{\frac{1}{k \Gamma_{k}(-\mu+m k)} \int_{0}^{z} f(t)(z-t)^{-\frac{\mu}{k}+m-1} d t\right\} .
\end{aligned}
$$

Theorem 5. In [25], let $k \in \mathbb{R}^{+}, \Re(\mu)<0$. Then, we have

$$
{ }_{k} \mathcal{D}_{z}^{\mu}\left\{z^{\frac{\eta}{k}}\right\}=\frac{z^{\frac{\eta-\mu}{k}}}{\Gamma_{k}(-\mu)} B_{k}(\eta+k,-\mu) .
$$

Theorem 6. In [25], let $\operatorname{Re}(\mu)>0$ and suppose that the function $f(z)$ is analytic at the origin with its Maclaurin expansion has the power series expansion

$$
f(z)=\sum_{n=0}^{\infty} a_{n} z^{n}
$$

where $|z|<\rho, \rho \in \mathbb{R}^{+}$. Then,

$$
{ }_{k} \mathcal{D}_{z}^{\mu}\{f(z)\}=\sum_{n=0}^{\infty} a_{n k} \mathcal{D}_{z}^{\mu}\left\{z^{n}\right\}
$$

Theorem 7. In [25], let $k \in \mathbb{R}^{+}, \Re(\mu)>\Re(\eta)>0$. Then, the following result holds true:

$$
{ }_{k} \mathcal{D}_{z}^{\eta-\mu}\left\{z^{\frac{\eta}{k}-1}(1-k z)^{-\frac{\beta}{k}}\right\}=\frac{\Gamma_{k}(\eta)}{\Gamma_{k}(\mu)} z^{\frac{\mu}{k}-1}{ }_{2} F_{1, k}\left[\begin{array}{c}
\beta, \eta \\
\mu
\end{array} ; z,\right.
$$

where $|z|<\frac{1}{k}$.

Theorem 8. In [25], let $k \in \mathbb{R}^{+}$. We have the following result:

$$
{ }_{k} \mathcal{D}_{z}^{\eta-\mu}\left\{z^{\frac{\eta}{k}-1}(1-k a z)^{-\frac{\alpha}{k}}(1-k b z)^{-\frac{\beta}{k}}\right\}=\frac{\Gamma_{k}(\eta)}{\Gamma_{k}(\mu)} z^{\frac{\mu}{k}-1} F_{1, k}(\eta, \alpha, \beta ; \mu ; a z, b z),
$$

where $\Re(\mu)>\Re(\eta)>0, \Re(\alpha)>0, \Re(\beta)>0$ and $\max \{|a z|,|b z|\}<\frac{1}{k}$.

\section{Transformation Formulas of $k$-Generalized Appell Functions}

In this section, we derive some linear transformations of $k$-generalized Appell functions and give some reduction formulas involving the ${ }_{2} F_{1, k}$ hypergeometric function which provide us with an opportunity to generalize widely used identities for Appell hypergeometric functions.

Theorem 9. For $k \in \mathbb{R}^{+}, F_{1, k}$ has the following relation:

$$
\begin{aligned}
& F_{1, k}\left(\alpha, \beta, \beta^{\prime} ; \gamma ; x, y\right) \\
= & (1-k x)^{-\frac{\beta}{k}}(1-k y)^{-\frac{\beta^{\prime}}{k}} F_{1, k}\left(\gamma-\alpha, \beta, \beta^{\prime} ; \gamma ;-\frac{x}{1-k x},-\frac{y}{1-k y}\right),
\end{aligned}
$$

where $\Re(\gamma)>\Re(\alpha)>0$ and $\left|\frac{x}{1-k x}\right|<\frac{1}{k},\left|\frac{y}{1-k y}\right|<\frac{1}{k},|x|<\frac{1}{k},|y|<\frac{1}{k}$. 
Proof. In [26], the integral representation of $F_{1, k}$ is given by

$$
F_{1, k}\left(\alpha, \beta, \beta^{\prime} ; \gamma ; x, y\right)=\frac{1}{k B_{k}(\alpha, \gamma-a)} \int_{0}^{1} t^{\frac{\alpha}{k}-1}(1-t)^{\frac{\gamma-\alpha}{k}-1}(1-k x t)^{-\frac{\beta}{k}}(1-k y t)^{-\frac{\beta^{\prime}}{k}} d t .
$$

If we make use of the substitution $t=1-t_{1}$ in the above integral, then we can write

$$
\begin{aligned}
& F_{1, k}\left(\alpha, \beta, \beta^{\prime} ; \gamma ; x, y\right)=\frac{1}{k B_{k}(\alpha, \gamma-a)} \\
& \times \int_{0}^{1} t_{1}^{\frac{\gamma-\alpha}{k}-1}\left(1-t_{1}\right)^{\frac{\alpha}{k}-1}\left(1-k x\left(1-t_{1}\right)\right)^{-\frac{\beta}{k}}\left(1-k y\left(1-t_{1}\right)\right)^{-\frac{\beta^{\prime}}{k}} d t_{1} \\
= & \frac{1}{k B_{k}(\alpha, \gamma-a)}(1-k x)^{-\frac{\beta}{k}}(1-k y)^{-\frac{\beta^{\prime}}{k}} \\
& \times \int_{0}^{1} t_{1}^{\frac{\gamma-\alpha}{k}-1}\left(1-t_{1}\right)^{\frac{\alpha}{k}-1}\left(1+\frac{k x t_{1}}{1-k x}\right)^{-\frac{\beta}{k}}\left(1+\frac{k y t_{1}}{1-k y}\right)^{-\frac{\beta^{\prime}}{k}} d t_{1} \\
= & (1-k x)^{-\frac{\beta}{k}}(1-k y)^{-\frac{\beta^{\prime}}{k}} F_{1, k}\left(\gamma-\alpha, \beta, \beta^{\prime} ; \gamma ;-\frac{x}{1-k x},-\frac{y}{1-k y}\right) .
\end{aligned}
$$

Thus, we get the desired result.

Theorem 10. For $k \in \mathbb{R}^{+}$, we have

$$
F_{1, k}\left(\alpha, \beta, \beta^{\prime} ; \gamma ; x, y\right)=(1-k x)^{-\frac{\alpha}{k}} F_{1, k}\left(\alpha, \gamma-\beta-\beta^{\prime}, \beta^{\prime} ; \gamma ;-\frac{x}{1-k x},-\frac{x-y}{1-k x}\right),
$$

and

$$
F_{1, k}\left(\alpha, \beta, \beta^{\prime} ; \gamma ; x, y\right)=(1-k y)^{-\frac{\alpha}{k}} F_{1, k}\left(\alpha, \beta, \gamma-\beta-\beta^{\prime} ; \gamma ;-\frac{y-x}{1-k y},-\frac{y}{1-k y}\right) .
$$

Proof. By a change of variables $t=\frac{t_{1}}{1-k x+k t_{1} x}$ in the integral representation of $F_{1, k}$, we have that

$$
\begin{aligned}
& F_{1, k}\left(\alpha, \beta, \beta^{\prime} ; \gamma ; x, y\right)=\frac{1}{k B_{k}(\alpha, \gamma-\alpha)} \\
& \times \int_{0}^{1} t^{\frac{\alpha}{k}-1}(1-t)^{\frac{\gamma-\alpha}{k}-1}(1-k x t)^{-\frac{\beta}{k}}(1-k y t)^{-\frac{\beta^{\prime}}{k}} d t \\
= & \frac{1}{k B_{k}(\alpha, \gamma-\alpha)}(1-k x)^{\frac{\gamma-\alpha-\beta}{k}} \\
& \times \int_{0}^{1} t_{1}^{\frac{\alpha}{k}-1}\left(1-t_{1}\right)^{\frac{\gamma-\alpha}{k}-1}\left(1-k x+k x t_{1}\right)^{\frac{\beta+\beta^{\prime}-\gamma}{k}}\left(1-k x+k x t_{1}-k y t_{1}\right)^{-\frac{\beta^{\prime}}{k}} d t_{1} \\
= & \frac{1}{k B_{k}(\alpha, \gamma-\alpha)}(1-k x)^{-\frac{\alpha}{k}} \\
& \times \int_{0}^{1} t_{1}^{\frac{\alpha}{k}-1}\left(1-t_{1}\right)^{\frac{\gamma-\alpha}{k}-1}\left(1+\frac{k x t_{1}}{1-k x}\right)^{\frac{\beta+\beta^{\prime}-\gamma}{k}}\left(1+\frac{k x t_{1}-k y t_{1}}{1-k x}\right)^{-\frac{\beta^{\prime}}{k}} d t_{1} \\
= & (1-k x)^{-\frac{\alpha}{k}} F_{1, k}\left(\alpha, \gamma-\beta-\beta^{\prime}, \beta^{\prime} ; \gamma ;-\frac{x}{1-k x},-\frac{x-y}{1-k x}\right) .
\end{aligned}
$$


In the above integral, we note that using a similar argument with $t=\frac{t_{1}}{1-k y-k t_{1} y}$, one can easily obtain

$$
F_{1, k}\left(\alpha, \beta, \beta^{\prime} ; \gamma ; x, y\right)=(1-k y)^{-\frac{\alpha}{k}} F_{1, k}\left(\alpha, \beta, \gamma-\beta-\beta^{\prime} ; \gamma ;-\frac{y-x}{1-k y},-\frac{y}{1-k y}\right) .
$$

Theorem 11. Letting $k \in \mathbb{R}^{+}$, then $F_{1, k}$ has the following relations:

$$
\begin{aligned}
& F_{1, k}\left(\alpha, \beta, \beta^{\prime} ; \gamma ; x, y\right) \\
= & (1-k x)^{\frac{\gamma-\alpha-\beta}{k}}(1-k y)^{-\frac{\beta^{\prime}}{k}} F_{1, k}\left(\gamma-\alpha, \gamma-\beta-\beta^{\prime}, \beta^{\prime} ; \gamma ; x,-\frac{y-x}{1-k y}\right), \\
& \text { and } \\
& F_{1, k}\left(\alpha, \beta, \beta^{\prime} ; \gamma ; x, y\right) \\
= & (1-k x)^{-\frac{\beta}{k}}(1-k y)^{\frac{\gamma-\alpha-\beta^{\prime}}{k}} F_{1, k}\left(\gamma-\alpha, \beta, \gamma-\beta-\beta^{\prime} ; \gamma ;-\frac{y-x}{1-k x}, y\right) .
\end{aligned}
$$

Proof. Using $t=\frac{t_{1}}{1-k x+k x t_{1}}$ and $t_{1}=1-t_{2}$ in integral representation of $F_{1, k}$, we obtain

$$
\begin{aligned}
& F_{1, k}\left(\alpha, \beta, \beta^{\prime} ; \gamma ; x, y\right)=\frac{1}{k B_{k}(\alpha, \gamma-\alpha)} \\
& \times \int_{0}^{1} t^{\frac{\alpha}{k}-1}(1-t)^{\frac{\gamma-\alpha}{k}-1}(1-k x t)^{-\frac{\beta}{k}}(1-k y t)^{-\frac{\beta^{\prime}}{k}} d t \\
= & \frac{1}{k B_{k}(\alpha, \gamma-\alpha)} \\
& \times \int_{0}^{1} t_{2}^{\frac{\gamma-\alpha}{k}-1}\left(1-t_{2}\right)^{\frac{\alpha}{k}-1}(1-k x)^{\frac{\gamma-\alpha-\beta}{k}}\left(1-k x t_{2}\right)^{\frac{\beta+\beta^{\prime}-\gamma}{k}}\left(1-k y+k y t_{2}-k x t_{2}\right)^{-\frac{\beta^{\prime}}{k}} d t_{2} \\
= & (1-k x)^{\frac{\gamma-\alpha-\beta}{k}}(1-k y)^{-\frac{\beta^{\prime}}{k}} \frac{1}{k B_{k}(\alpha, \gamma-\alpha)} \\
& \times \int_{0}^{1} t_{2}^{\frac{\gamma-\alpha}{k}-1}\left(1-t_{2}\right)^{\frac{\alpha}{k}-1}\left(1-k x t_{2}\right)^{\frac{\beta+\beta^{\prime}-\gamma}{k}}\left(1+\frac{k y t_{2}-k x t_{2}}{1-k y}\right)^{-\frac{\beta^{\prime}}{k}} d t_{2} \\
= & (1-k x)^{\frac{\gamma-\alpha-\beta}{k}}(1-k y)^{-\frac{\beta^{\prime}}{k}} F_{1, k}\left(\gamma-\alpha, \gamma-\beta-\beta^{\prime}, \beta^{\prime} ; \gamma ; x,-\frac{y-x}{1-k y}\right) .
\end{aligned}
$$

Using the same method as above, we can reach (45) easily. 
Theorem 12. Letting $k \in \mathbb{R}^{+}$, then the following relations hold:

$$
\begin{aligned}
& F_{2, k}\left(\alpha, \beta, \beta^{\prime} ; \gamma, \gamma^{\prime} ; x, y\right) \\
= & (1-k x)^{-\frac{\alpha}{k}} F_{2, k}\left(\alpha, \gamma-\beta, \beta^{\prime} ; \gamma, \gamma^{\prime} ;-\frac{x}{1-k x}, \frac{y}{1-k x}\right), \\
& F_{2, k}\left(\alpha, \beta, \beta^{\prime} ; \gamma, \gamma^{\prime} ; x, y\right) \\
= & (1-k y)^{-\frac{\alpha}{k}} F_{2, k}\left(\alpha, \beta, \gamma^{\prime}-\beta^{\prime} ; \gamma, \gamma^{\prime} ; \frac{x}{1-k y},-\frac{y}{1-k y}\right), \\
& \text { and } \\
& F_{2, k}\left(\alpha, \beta, \beta^{\prime} ; \gamma, \gamma^{\prime} ; x, y\right) \\
= & (1-k x-k y)^{-\frac{\alpha}{k}} F_{2, k}\left(\alpha, \gamma-\beta, \gamma^{\prime}-\beta^{\prime} ; \gamma, \gamma^{\prime} ;-\frac{x}{1-k x-k y},-\frac{y}{1-k x-k y}\right) .
\end{aligned}
$$

Proof. By taking for the first relation $t=1-t_{1}$, for the second $s=1-s_{1}$ and, finally, for the third $t=1-t_{1}, s=1-s_{1}$ together in the double integral (30), we find (46), (47) and (48), respectively. These complete the proof.

We continue with some reduction formulas for Appell functions $F_{1, k}$ and $F_{2, k}$ in terms of the ${ }_{2} F_{1, k}$ generalized hypergeometric function.

Theorem 13. Let $k \in \mathbb{R}^{+}$. Then, the special cases of $F_{1, k}$ and $F_{2, k}$ are as follows:

$$
\begin{aligned}
F_{1, k}\left(\alpha, \beta, \beta^{\prime} ; \gamma ; x, y\right) & =(1-k x)^{-\frac{\alpha}{k}}{ }_{2} F_{1, k}\left[\begin{array}{c}
\alpha, \beta^{\prime} \\
\beta+\beta^{\prime} ;-\frac{x-y}{1-k x}
\end{array}\right], \\
F_{1, k}\left(\alpha, \beta, \beta^{\prime} ; \gamma ; x, y\right) & =(1-k y)^{-\frac{\alpha}{k}}{ }_{2} F_{1, k}\left[\begin{array}{c}
\alpha, \beta \\
\beta+\beta^{\prime} ;-\frac{y-x}{1-k y}
\end{array}\right], \\
F_{2, k}\left(\alpha, \beta, \beta^{\prime} ; \gamma, \gamma^{\prime} ; x, y\right) & =(1-k x)^{-\frac{\alpha}{k}}{ }_{2} F_{1, k}\left[\begin{array}{c}
\alpha, \beta^{\prime} \\
\gamma^{\prime} ; \frac{y}{1-k x}
\end{array}\right], \\
F_{2, k}\left(\alpha, \beta, \beta^{\prime} ; \gamma, \gamma^{\prime} ; x, y\right) & =(1-k y)^{-\frac{\alpha}{k}}{ }_{2} F_{1, k}\left[\begin{array}{c}
\alpha, \beta \\
\gamma
\end{array} ; \frac{x}{1-k y}\right] .
\end{aligned}
$$

Proof. Specializing (42) and (43) for $\gamma=\beta+\beta^{\prime}$ and also if we set $\gamma=\beta$ and $\gamma=\beta^{\prime}$ in (46) and (47), we obtain desired results, respectively.

In the next lemma, we will prove Euler transformation for ${ }_{2} F_{1, k}$ hypergeometric function, which will be used in the next theorem.

Lemma 1. Let $x \in \mathbb{C}, k \in \mathbb{R}^{+}$. Then, we have

$$
{ }_{2} F_{1, k}\left[\begin{array}{c}
\alpha, \beta \\
\gamma
\end{array} ; x\right]=(1-k x)^{-\frac{\beta}{k}}{ }_{2} F_{1, k}\left[\begin{array}{c}
\gamma-\alpha, \beta \\
\gamma
\end{array} ;-\frac{x}{1-k x}\right] .
$$


Proof. From the definition of ${ }_{2} F_{1, k}$, one gets

$$
\begin{aligned}
& (1-k x)^{-\frac{\beta}{k}}{ }_{2} F_{1, k}\left[\begin{array}{c}
\gamma-\alpha, \beta \\
\gamma
\end{array}-\frac{x}{1-k x}\right] \\
= & (1-k x)^{-\frac{\beta}{k}} \sum_{n=0}^{\infty} \frac{(\gamma-\alpha)_{n, k}(\beta)_{n, k}}{(\gamma)_{n, k}} \frac{(-1)^{n} x^{n}}{n !(1-k x)^{n}} \\
= & \sum_{m, n=0}^{\infty} \frac{(\gamma-\alpha)_{n, k}(\beta)_{n, k}(\beta+n k)_{m, k}}{(\gamma)_{n, k}} \frac{(-1)^{n} x^{m+n}}{n ! m !} \\
= & \sum_{m=0}^{\infty} \sum_{n=0}^{m} \frac{(\gamma-\alpha)_{n, k}(\beta)_{m, k}}{(\gamma)_{n, k}} \frac{(-1)^{n} x^{m}}{n !(m-n) !} .
\end{aligned}
$$

Using the identity $(m-n) !=\frac{(-1)^{n} m !}{(-m)_{n}}$ in (54), we thus find that

$$
\begin{aligned}
& (1-k x)^{-\frac{\beta}{k}}{ }_{2} F_{1, k}\left[\begin{array}{c}
\gamma-\alpha, \beta ;-\frac{x}{1-k x} \\
\gamma
\end{array}\right] \\
= & \sum_{m=0}^{\infty} \sum_{n=0}^{m} \frac{(\gamma-\alpha)_{n, k}(-m)_{n}}{(\gamma)_{n, k} n !} \frac{(\beta)_{m, k} x^{m}}{m !} \\
= & \sum_{m=0}^{\infty}{ }_{2} F_{1, k}\left[\begin{array}{c}
(-m, 1),(\gamma-\alpha, k) \\
(\gamma, k)
\end{array} 1\right](\beta)_{m, k} \frac{x^{m}}{m !} .
\end{aligned}
$$

Making use of (24) in (55), we get the desired result.

Theorem 14. Let $k \in \mathbb{R}^{+}$. Then, we have

$$
F_{1, k}\left(\alpha, \beta, \beta^{\prime} ; \gamma ; x, y\right)=(1-k y)^{-\frac{\beta^{\prime}}{k}} F_{3, k}\left(\alpha, \gamma-\alpha, \beta, \beta^{\prime} ; \gamma ; x,-\frac{y}{1-k y}\right)
$$

Proof. Using the definition of $F_{1, k}$ defined by (25) and making use of (53), we can write

$$
\begin{aligned}
F_{1, k}\left(\alpha, \beta, \beta^{\prime} ; \gamma ; x, y\right) & =\sum_{m=0}^{\infty} \frac{(\alpha)_{m, k}(\beta)_{m, k}}{(\gamma)_{m, k}}{ }_{2} F_{1, k}\left[\begin{array}{c}
\alpha+m k, \beta^{\prime} \\
\gamma+m k
\end{array} ; y\right] \frac{x^{m}}{m !} \\
& =\sum_{m=0}^{\infty} \frac{(\alpha)_{m, k}(\beta)_{m, k}}{(\gamma)_{m, k}}(1-k y)^{-\frac{\beta^{\prime}}{k}}{ }_{2} F_{1, k}\left[\begin{array}{c}
\beta^{\prime}, \gamma-\alpha \\
\gamma+m k
\end{array} ;-\frac{y}{1-k y}\right] \frac{x^{m}}{m !} \\
& =(1-k y)^{-\frac{\beta^{\prime}}{k}} \sum_{m, n=0}^{\infty} \frac{(\alpha)_{m, k}(\beta)_{m, k}\left(\beta^{\prime}\right)_{n, k}(\gamma-\alpha)_{n, k}}{(\gamma)_{m, k}(\gamma+m k)_{n, k}} \frac{x^{m}}{m !} \frac{\left(-\frac{y}{1-k y}\right)^{n}}{n !} \\
& =(1-k y)^{-\frac{\beta^{\prime}}{k}} F_{3, k}\left(\alpha, \gamma-\alpha, \beta, \beta^{\prime} ; \gamma ; x,-\frac{y}{1-k y}\right) .
\end{aligned}
$$

Thus, we finish the proof.

\section{Generating Relations Involving the Generalized Appell Functions}

In this section, employing the theory of Riemann-Liouville $k$-fractional derivative [25] and making use of the relations which we have considered in the previous sections, we establish linear and bilinear generating relations for $k$-analogue of hypergeometric functions and $k$-Appell functions. 
Theorem 15. We have the generating relation

$$
\sum_{n=0}^{\infty} \frac{(\lambda)_{n, k}}{n !}{ }_{2} F_{1, k}\left[\begin{array}{cc}
\lambda+n k, & \alpha \\
\beta & ; x
\end{array}\right] t^{n}=(1-k t)^{-\frac{\lambda}{k}}{ }_{2} F_{1, k}\left[\begin{array}{cc}
\lambda, & \alpha \\
\beta & ; \frac{x}{1-k t}
\end{array}\right],
$$

where $|x|<\frac{1}{k} \min \{1,1-k t\}$.

Proof. To prove the result, consider the elementary identities given by

$$
\begin{aligned}
& (1-k x-k t)^{-\frac{\lambda}{k}}=(1-k t)^{-\frac{\lambda}{k}}\left(1-\frac{k x}{1-k t}\right)^{-\frac{\lambda}{k}}, \\
& (1-k x-k t)^{-\frac{\lambda}{k}}=(1-k x)^{-\frac{\lambda}{k}}\left(1-\frac{k t}{1-k x}\right)^{-\frac{\lambda}{k}} .
\end{aligned}
$$

From the series expansion using the definition of the Pochhammer $k$-symbol [13]

$$
\sum_{n=0}^{\infty}(\alpha)_{n, k} \frac{z^{n}}{n !}=(1-k z)^{-\frac{\alpha}{k}}
$$

We can write

$$
\begin{aligned}
(1-k x-k t)^{-\frac{\lambda}{k}} & =(1-k x)^{-\frac{\lambda}{k}} \sum_{n=0}^{\infty} \frac{(\lambda)_{n, k}}{n !}\left(\frac{t}{1-k x}\right)^{n} \\
& =(1-k x)^{-\frac{\lambda}{k}} \sum_{n=0}^{\infty} \frac{(\lambda)_{n, k}}{n !}(1-k x)^{-n} t^{n} \\
& =\sum_{n=0}^{\infty} \frac{(\lambda)_{n, k}}{n !}(1-k x)^{-\frac{\lambda}{k}-n} t^{n} .
\end{aligned}
$$

From (58) and (59), we have the equality

$$
\sum_{n=0}^{\infty} \frac{(\lambda)_{n, k}}{n !}(1-k x)^{-\frac{\lambda}{k}-n} t^{n}=(1-k t)^{-\frac{\lambda}{k}}\left(1-\frac{k x}{1-k t}\right)^{-\frac{\lambda}{k}}
$$

where $|t|<|1-k x|$. Multiplying both sides of (60) by $x^{\frac{\alpha}{k}-1}$ and then applying ${ }_{k} D_{x}^{\alpha-\beta}$ to both sides of $(60)$, we can reach

$$
{ }_{k} D_{x}^{\alpha-\beta}\left\{\sum_{n=0}^{\infty} \frac{(\lambda)_{n, k}}{n !} x^{\frac{\alpha}{k}-1}(1-k x)^{-\frac{\lambda}{k}-n} t^{n}\right\}={ }_{k} D_{x}^{\alpha-\beta}\left\{(1-k t)^{-\frac{\lambda}{k}} x^{\frac{\alpha}{k}-1}\left(1-\frac{k x}{1-k t}\right)^{-\frac{\lambda}{k}}\right\} .
$$

Since $\Re(\alpha)>0$ where $|t|<|1-k x|$, it is possible to change the order of the summation and differentiation, we get

$$
\begin{aligned}
& \sum_{n=0}^{\infty} \frac{(\lambda)_{n, k}}{n !}{ }_{k} D_{x}^{\alpha-\beta}\left\{x^{\frac{\alpha}{k}-1}(1-k x)^{-\frac{\lambda}{k}-n}\right\} t^{n} \\
= & (1-k t)^{-\frac{\lambda}{k}}{ }_{k} D_{x}^{\alpha-\beta}\left\{x^{\frac{\alpha}{k}-1}\left(1-\frac{k x}{1-k t}\right)^{-\frac{\lambda}{k}}\right\} .
\end{aligned}
$$


Finally, using relation (39) in (61), it follows that

$$
\sum_{n=0}^{\infty} \frac{(\lambda)_{n, k}}{n !}{ }_{2} F_{1, k}\left[\begin{array}{cc}
\lambda+n k, & \alpha \\
\beta & ; x
\end{array}\right] t^{n}=(1-k t)^{-\frac{\lambda}{k}}{ }_{2} F_{1, k}\left[\begin{array}{cc}
\lambda, & \alpha \\
\beta & ; \frac{x}{1-k t}
\end{array}\right],
$$

where $|x|<\frac{1}{k} \min \{1,1-k t\}$. Hence, we get the desired result.

Theorem 16. We have the generating relation

$$
\begin{aligned}
& \sum_{n=0}^{\infty} \frac{(\lambda)_{n, k}}{n !}{ }_{2} F_{1, k}\left[\begin{array}{cc}
\rho-n k, & \alpha \\
\beta & ; x
\end{array}\right] t^{n} \\
= & (1-k t)^{-\frac{\lambda}{k}} F_{1, k}\left[\alpha, \rho, \lambda ; \beta ; x,-\frac{k x t}{1-k t}\right],
\end{aligned}
$$

where $|x|<\frac{1}{k},\left|\frac{k x t}{1-k t}\right|<\frac{1}{k}$.

Proof. Consider the identity

$$
(1-k(1-k x) t)^{-\frac{\lambda}{k}}=(1-k t)^{-\frac{\lambda}{k}}\left(1+\frac{k^{2} x t}{1-k t}\right)^{-\frac{\lambda}{k}}
$$

Under the assumption $|k t|<|1-k x|^{-1}$,we can rewrite (63)

$$
\sum_{n=0}^{\infty} \frac{(\lambda)_{n, k}}{n !}(1-k x)^{n} t^{n}=(1-k t)^{-\frac{\lambda}{k}}\left(1+\frac{k^{2} x t}{1-k t}\right)^{-\frac{\lambda}{k}}
$$

Multiplying $x^{\frac{\alpha}{k}-1}(1-k x)^{-\frac{\rho}{k}}$ and taking the ${ }_{k} D_{x}^{\alpha-\beta}$ on both sides of (64), we obtain

$$
\begin{aligned}
& { }_{k} D_{x}^{\alpha-\beta}\left\{\sum_{n=0}^{\infty} \frac{(\lambda)_{n, k}}{n !} x^{\frac{\alpha}{k}-1}(1-k x)^{n-\frac{\rho}{k}} t^{n}\right\} \\
= & { }_{k} D_{x}^{\alpha-\beta}\left\{x^{\frac{\alpha}{k}-1}(1-k t)^{-\frac{\lambda}{k}}(1-k x)^{-\frac{\rho}{k}}\left(1+k \frac{k x t}{1-k t}\right)^{-\frac{\lambda}{k}}\right\} .
\end{aligned}
$$

For $\Re(\alpha)>0$, interchanging the order of the summation and the operator ${ }_{k} D_{x}^{\alpha-\beta}$, we have

$$
\begin{aligned}
& \sum_{n=0}^{\infty} \frac{(\lambda)_{n, k}}{n !}{ }_{k} D_{x}^{\alpha-\beta}\left\{x^{\frac{\alpha}{k}-1}(1-k x)^{n-\frac{\rho}{k}}\right\} t^{n} \\
= & (1-k t)^{-\frac{\lambda}{k}}{ }_{k} D_{x}^{\alpha-\beta}\left\{x^{\frac{\alpha}{k}-1}(1-k x)^{-\frac{\rho}{k}}\left(1+k \frac{k x t}{1-k t}\right)^{-\frac{\lambda}{k}}\right\} .
\end{aligned}
$$

Assuming $|x|<\frac{1}{k}$ and $\left|\frac{k x t}{1-k t}\right|<\frac{1}{k}$ and using (39) and (40),

$$
\sum_{n=0}^{\infty} \frac{(\lambda)_{n, k}}{n !}{ }_{2} F_{1, k}\left[\begin{array}{cc}
\rho-n k, & \alpha \\
\beta &
\end{array}\right] x t^{n}=(1-k t)^{-\frac{\lambda}{k}} F_{1, k}\left[\alpha, \rho, \lambda ; \beta ; x,-\frac{k x t}{1-k t}\right]
$$

the theorem is immediate. 
Theorem 17. We have the generating relations

$$
\begin{aligned}
& \sum_{n=0}^{\infty} \frac{(\beta-\rho)_{n, k}}{n !}{ }_{2} F_{1, k}\left[\begin{array}{cc}
\rho-n k, & \alpha \\
\beta & ; x
\end{array}\right] t^{n} \\
= & (1-k t)^{\frac{\alpha+\rho-\beta}{k}}\left(1-k t+k^{2} x t\right)^{-\frac{\alpha}{k}}{ }_{2} F_{1, k}\left[\begin{array}{cc}
\alpha, \rho \\
\beta
\end{array} ; \frac{x}{1-k t+k^{2} x t}\right], \\
& \text { and } \\
& \sum_{n=0}^{\infty} \frac{(\beta)_{n, k}(\gamma)_{n, k}}{(\delta)_{n, k} n !}{ }_{2} F_{1, k}\left[\begin{array}{rr}
-n k, & \alpha \\
\beta &
\end{array}\right] t^{n} \\
= & F_{1, k}(\gamma, \beta-\alpha, \alpha ; \delta ; t,(1-k x) t) .
\end{aligned}
$$

Proof. We use the result of the previous theorem. Setting $\lambda=\beta-\rho$ in (62), we find that

$$
\sum_{n=0}^{\infty} \frac{(\beta-\rho)_{n, k}}{n !}{ }_{2} F_{1, k}\left[\begin{array}{cc}
\rho-n k, & \alpha \\
\beta & ; x
\end{array}\right] t^{n}=(1-k t)^{\frac{\rho-\beta}{k}} F_{1, k}\left[\alpha, \rho, \beta-\rho ; \beta ; x,-\frac{k x t}{1-k t}\right] .
$$

If we use the reduction formula for $F_{1, k}$ given by (50), we can easily obtain the desired result as follows:

$$
\begin{aligned}
& \sum_{n=0}^{\infty} \frac{(\beta-\rho)_{n, k}}{n !}{ }_{2} F_{1, k}\left[\begin{array}{cc}
\rho-n k, & \alpha \\
\beta & ; x
\end{array}\right] t^{n} \\
= & (1-k t)^{\frac{\alpha+\rho-\beta}{k}}\left(1-k t+k^{2} x t\right)^{-\frac{\alpha}{k}}{ }_{2} F_{1, k}\left[\begin{array}{c}
\alpha, \rho \\
\beta
\end{array} ; \frac{x}{1-k t+k^{2} x t}\right] .
\end{aligned}
$$

For $\rho=0,(67)$ gives

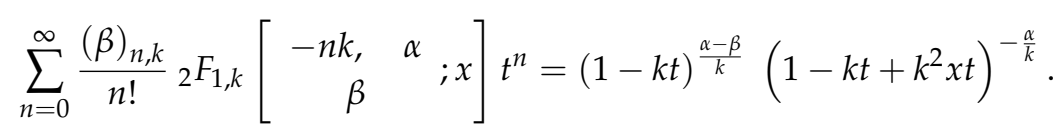

Multiplying both sides of (68) with $t^{\frac{\gamma}{k}-1}$ and operation of the ${ }_{k} D_{t}^{\gamma-\delta}$ on (68), one can easily obtain

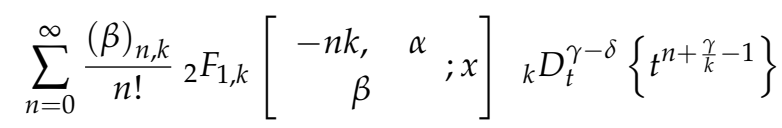

$$
\begin{aligned}
& ={ }_{k} D_{t}^{\gamma-\delta}\left\{t^{\frac{\gamma}{k}-1}(1-k t)^{\frac{\alpha-\beta}{k}}\left(1-k t+k^{2} x t\right)^{-\frac{\alpha}{k}}\right\} \text {. }
\end{aligned}
$$

In view of (36) and (40) on the right and left sides of (69), respectively, we can reach

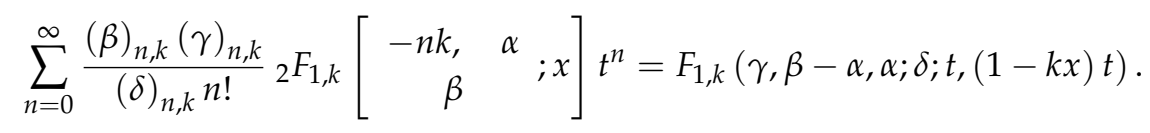

Theorem 18. We have the generating relation

$$
\begin{aligned}
& \sum_{n=0}^{\infty} \frac{(\lambda)_{n, k}}{n !}{ }_{2} F_{1, k}\left[\begin{array}{cc}
\lambda+n k, & \alpha \\
\beta & ; x
\end{array}\right]{ }_{2} F_{1, k}\left[\begin{array}{rr}
-n k, & \gamma \\
\delta & ; y
\end{array}\right] t^{n} \\
= & (1-k t)^{-\frac{\lambda}{k}} F_{2, k}\left(\lambda, \alpha, \gamma ; \beta, \delta ; \frac{x}{1-k t},-\frac{k y t}{1-k t}\right) .
\end{aligned}
$$


Proof. Putting $(1-k y) t$ instead of $t$ in (57), we can obtain

$$
\begin{aligned}
& \sum_{n=0}^{\infty} \frac{(\lambda)_{n, k}}{n !}{ }_{2} F_{1, k}\left[\begin{array}{cc}
\lambda+n k, & \alpha \\
\beta &
\end{array}\right]\left[(1-k y)^{n} t^{n}\right.
\end{aligned}
$$

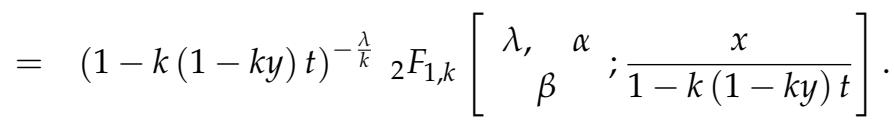

Multiplying with $y^{\frac{\gamma}{k}-1}$, employing ${ }_{k} D_{y}^{\gamma-\delta}$ both sides of (71) and the under the assumption $\Re(\gamma)>0$ interchanging differentiation and summation, we can write

$$
\begin{aligned}
& \sum_{n=0}^{\infty} \frac{(\lambda)_{n, k}}{n !}{ }_{2} F_{1, k}\left[\begin{array}{cc}
\lambda+n k, & \alpha \\
\beta & ; x
\end{array}\right]{ }_{k} D_{y}^{\gamma-\delta}\left\{y^{\frac{\gamma}{k}-1}(1-k y)^{n}\right\} t^{n} \\
= & { }_{k} D_{y}^{\gamma-\delta}\left\{y^{\frac{\gamma}{k}-1}(1-k(1-k y) t)^{-\frac{\lambda}{k}}{ }_{2} F_{1, k}\left[\begin{array}{cc}
\lambda, & \alpha \\
\beta & ; \frac{x}{1-k(1-k y) t}
\end{array}\right]\right\} .
\end{aligned}
$$

Make use of the formula (39), we can easily simplify left side of the (72) as follows:

$$
\begin{aligned}
& \sum_{n=0}^{\infty} \frac{(\lambda)_{n, k}}{n !}{ }_{2} F_{1, k}\left[\begin{array}{cc}
\lambda+n k, & \alpha \\
\beta & ; x
\end{array}\right]{ }_{k} D_{y}^{\gamma-\delta}\left\{y^{\frac{\gamma}{k}-1}(1-k y)^{n}\right\} t^{n}
\end{aligned}
$$

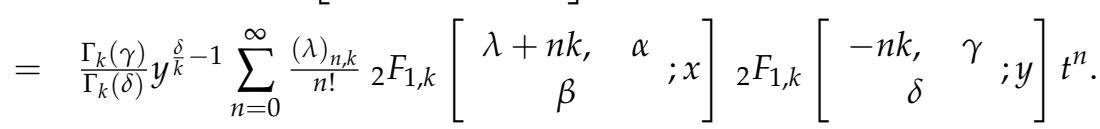

For the right side of the (72), using the definition of ${ }_{2} F_{1, k}$ and the formula (36), one can obtain

$$
\begin{aligned}
& { }_{k} D_{y}^{\gamma-\delta}\left\{y^{\frac{\gamma}{k}-1}(1-k(1-k y) t)^{-\frac{\lambda}{k}}{ }_{2} F_{1, k}\left[\begin{array}{c}
\lambda, \\
\beta
\end{array} ; \frac{x}{1-k(1-k y) t}\right]\right\} \\
= & \frac{\Gamma_{k}(\gamma)}{\Gamma_{k}(\delta)}(1-k t)^{-\frac{\lambda}{k}} y^{\frac{\delta}{k}-1} F_{2, k}\left(\lambda, \alpha, \gamma ; \beta, \delta ; \frac{x}{1-k t^{\prime}},-\frac{k y t}{1-k t}\right),
\end{aligned}
$$

where $|x|<\frac{1}{k},|y|<\frac{1}{k},\left|\frac{x}{1-k t}\right|+\left|\frac{k y t}{1-k t}\right|<\frac{1}{k},\left|\frac{1-k y}{1-x} t\right|<\frac{1}{k}$. Combining the relations (73) and (74), we get desired result.

As a special case of (70), we give the following theorem.

Theorem 19. We have the generating relation

$$
\begin{aligned}
& \sum_{n=0}^{\infty} \frac{(\beta-\rho)_{n, k}}{n !}{ }_{2} F_{1, k}\left[\begin{array}{cc}
\rho-n k, & \alpha \\
\beta & ; x
\end{array}\right]{ }_{2} F_{1, k}\left[\begin{array}{rr}
-n k, & \gamma \\
\delta & ; y
\end{array}\right] t^{n} \\
= & (1-k x)^{-\frac{\alpha}{k}}(1-k t)^{\frac{\rho-\beta}{k}} F_{2, k}\left(\beta-\rho, \alpha, \gamma ; \beta, \delta ;-\frac{x}{(1-k x)(1-k t)},-\frac{k y t}{1-k t}\right) .
\end{aligned}
$$

Proof. For $\lambda=\beta-\rho$ in (70), we get

$$
\begin{aligned}
& \sum_{n=0}^{\infty} \frac{(\beta-\rho)_{n, k}}{n !}{ }_{2} F_{1, k}\left[\begin{array}{cc}
\beta-\rho+n k, & \alpha \\
\beta & ; x
\end{array}\right]{ }_{2} F_{1, k}\left[\begin{array}{rr}
-n k, & \gamma ; y \\
\delta &
\end{array}\right] t^{n} \\
= & (1-k t)^{\frac{\rho-\beta}{k}} F_{2, k}\left(\beta-\rho, \alpha, \gamma ; \beta, \delta ; \frac{x}{1-k t},-\frac{k y t}{1-k t}\right) .
\end{aligned}
$$


Using Euler transformation given by (53) for ${ }_{2} F_{1, k}$

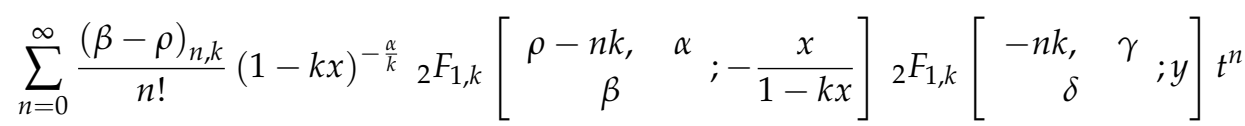

$$
\begin{aligned}
& =(1-k t)^{\frac{\rho-\beta}{k}} F_{2, k}\left(\beta-\rho, \alpha, \gamma ; \beta, \delta ; \frac{x}{1-k t},-\frac{k y t}{1-k t}\right) \text {, }
\end{aligned}
$$

and putting $-\frac{x}{1-k x}$ instead of $x$, we reach the desired result.

Theorem 20. We have the generating relation

$$
\begin{aligned}
& \sum_{n=0}^{\infty} \frac{(\lambda)_{n, k}}{n !}{ }_{2} F_{1, k}\left[\begin{array}{cc}
\lambda+n k, & \alpha \\
\beta & ; x
\end{array}\right]{ }_{2} F_{1, k}\left[\begin{array}{cc}
\lambda+n k, & \gamma \\
\delta & ; y
\end{array}\right] t^{n} \\
= & (1-k t)^{-\frac{\lambda}{k}} \sum_{n=0}^{\infty} \frac{(\lambda)_{n, k}(\alpha)_{n, k}}{(\beta)_{n, k} n !}\left(-\frac{k x y}{1-k t}\right)^{n} \\
& \times F_{2, k}\left(\lambda+n k, \alpha+n k, \gamma+n k ; \beta+n k, \delta+n k ; \frac{x}{1-k t},-\frac{k y}{1-k t}\right) .
\end{aligned}
$$

Proof. Replacing $t$ by $\frac{t}{1-k y}$ and after some simplification in (57), we find that

$$
\begin{aligned}
& \sum_{n=0}^{\infty} \frac{(\lambda)_{n, k}}{n !}{ }_{2} F_{1, k}\left[\begin{array}{cc}
\lambda+n k, & \alpha \\
\beta & ; x
\end{array}\right] \frac{t^{n}}{(1-k y)^{n+\frac{\lambda}{k}}} \\
& =(1-k t)^{-\frac{\lambda}{k}} \sum_{n=0}^{\infty} \frac{(\lambda)_{n, k}(\alpha)_{n, k}}{(\beta)_{n, k} n !}\left(\frac{x(1-k y)}{1-k t}\right)^{n}\left(1-\frac{k y}{1-k t}\right)^{-n-\frac{\lambda}{k}} \text {. }
\end{aligned}
$$

Using the binomial expansion $(x+y)^{n}=\sum_{k=0}^{n}\left(\begin{array}{l}n \\ k\end{array}\right) x^{k} y^{n-k}$,

$$
\begin{aligned}
& \sum_{n=0}^{\infty} \frac{(\lambda)_{n, k}}{n !}{ }_{2} F_{1, k}\left[\begin{array}{cc}
\lambda+n k, & \alpha \\
\beta & ; x
\end{array}\right] \frac{t^{n}}{(1-k y)^{n+\frac{\lambda}{k}}} \\
& =(1-k t)^{-\frac{\lambda}{k}} \\
& \times \sum_{n=0}^{\infty} \sum_{k_{1}=0}^{n} \frac{(\lambda)_{n, k}(\alpha)_{n, k}}{(\beta)_{n, k} n !}\left(\begin{array}{c}
n \\
k_{1}
\end{array}\right)(-1)^{n-k_{1}}\left(\frac{x}{1-k t}\right)^{k_{1}}\left(\frac{x k y}{1-k t}\right)^{n-k_{1}}\left(1-\frac{k y}{1-k t}\right)^{-n-\frac{\lambda}{k}} \\
& =(1-k t)^{-\frac{\lambda}{k}} \\
& \times \sum_{n, k_{1}=0}^{\infty} \frac{(\lambda)_{n+k_{1}, k}(\alpha)_{n+k_{1}, k}}{(\beta)_{n+k_{1}, k}\left(n+k_{1}\right) !}\left(\begin{array}{c}
n+k_{1} \\
k_{1}
\end{array}\right)(-1)^{n}\left(\frac{x}{1-k t}\right)^{k_{1}}\left(\frac{x k y}{1-k t}\right)^{n}\left(1-\frac{k y}{1-k t}\right)^{-n-k_{1}-\frac{\lambda}{k}} \\
& =(1-k t)^{-\frac{\lambda}{k}} \\
& \times \sum_{n=0}^{\infty} \frac{(\lambda)_{n, k}(\alpha)_{n, k}}{(\beta)_{n, k} n !}\left(-\frac{x k y}{1-k t}\right)^{n}\left(1-\frac{k y}{1-k t}\right)^{-n-\frac{\lambda}{k}}{ }_{2} F_{1, k}\left[\begin{array}{c}
\lambda+n k, \alpha+n k \\
\beta+n k
\end{array} \frac{\frac{x}{1-k t}}{1-\frac{k y}{1-k t}}\right](77)
\end{aligned}
$$

Multiplying $y^{\frac{\gamma}{k}-1}$, operating ${ }_{k} D_{y}^{\gamma-\delta}$ and applying (36), (39), and (40) together both sides of the (77) (in a similar way of proof of the (70)) for $|x|<\frac{1}{k},|y|<\frac{1}{k},\left|\frac{x}{1-k t}\right|+\left|\frac{k y}{1-k t}\right|<\frac{1}{k}$, we complete the proof. 
Theorem 21. We have the generating relation

$$
\begin{aligned}
& \sum_{n=0}^{\infty} \frac{(\lambda)_{n, k}}{n !}{ }_{2} F_{1, k}\left[\begin{array}{cc}
\lambda+n k, & \alpha \\
\beta & ; x
\end{array}\right]{ }_{2} F_{1, k}\left[\begin{array}{cc}
\lambda+n k, & \gamma \\
\delta & ; y
\end{array}\right] t^{n} \\
= & (1-k t)^{-\frac{\lambda}{k}} \sum_{n=0}^{\infty} \frac{(\lambda)_{n, k}(\alpha)_{n, k}(\gamma)_{n, k}}{(\beta)_{n, k}(\delta)_{n, k} n !}\left(\frac{k^{3} x y t}{(1-k t)^{2}}\right)^{n} \\
& \times{ }_{2} F_{1, k}\left[\begin{array}{c}
\lambda+n k, \alpha+n k \\
\beta+n k
\end{array} \frac{x}{1-k t}\right]{ }_{2} F_{1, k}\left[\begin{array}{c}
\lambda+n k, \\
\delta+n k
\end{array} ; \frac{y}{1-k t}\right] .
\end{aligned}
$$

For the special case $\beta=\delta=\lambda$, we have

$$
\begin{aligned}
& \sum_{n=0}^{\infty} \frac{(\lambda)_{n, k}}{n !}{ }_{2} F_{1, k}\left[\begin{array}{cc}
\lambda+n k, & \alpha \\
\lambda & ; x
\end{array}\right]{ }_{2} F_{1, k}\left[\begin{array}{rr}
\lambda+n k, & \gamma \\
\lambda & ; y
\end{array}\right] t^{n} \\
= & (1-k t)^{\frac{\gamma+\alpha-\lambda}{k}}(1-k t-k x)^{-\frac{\alpha}{k}}(1-k t-k y)^{-\frac{\gamma}{k}} \\
& \times{ }_{2} F_{1, k}\left[\begin{array}{c}
\alpha, \\
\lambda
\end{array} ; \frac{k^{3} x y t}{(1-k t-k x)(1-k t-k y)}\right]
\end{aligned}
$$

Proof. From the elementary identity, we find that

$$
((1-k x)(1-k y)-k t)^{-\frac{\lambda}{k}}=(1-k t)^{-\frac{\lambda}{k}}\left(\left(1-\frac{k x}{1-k t}\right)\left(1-\frac{k y}{1-k t}\right)-\frac{k^{3} x y t}{(1-k t)^{2}}\right)^{-\frac{\lambda}{k}}
$$

for $\left|\frac{k t}{(1-k x)(1-k y)}\right|<\frac{1}{k}$ and $\left|\frac{k^{3} x y t}{(1-k t-k x)(1-k t-k y)}\right|<\frac{1}{k}$. Applying (20) to (80), multiplying $x^{\frac{\alpha}{k}-1} y^{\frac{\gamma}{k}-1}$ and taking ${ }_{k} D_{x}^{\alpha-\beta}{ }_{k} D_{y}^{\gamma-\delta}$ together both sides of (80), we have

$$
\begin{aligned}
& { }_{k} D_{x}^{\alpha-\beta}{ }_{k} D_{y}^{\gamma-\delta}\left\{\sum_{n=0}^{\infty} \frac{(\lambda)_{n, k}}{n !} x^{\frac{\alpha}{k}-1}(1-k x)^{-\frac{\lambda}{k}-n} y^{\frac{\gamma}{k}-1}(1-k y)^{-\frac{\lambda}{k}-n} t^{n}\right\} \\
= & (1-k t)^{-\frac{\lambda}{k}} \\
& \times{ }_{k} D_{x}^{\alpha-\beta}{ }_{k} D_{y}^{\gamma-\delta}\left\{\sum_{n=0}^{\infty} \frac{(\lambda)_{n, k}\left(k^{3} t\right)^{n}}{n !(1-k t)^{2 n}} x^{\frac{\alpha}{k}+n-1}\left(1-\frac{k x}{1-k t}\right)^{-\frac{\lambda}{k}-n} y^{\frac{\gamma}{k}+n-1}\left(1-\frac{k y}{1-k t}\right)^{-\frac{\lambda}{k}-n}\right\} .
\end{aligned}
$$

Under the conditions $\Re(\alpha)>0, \Re(\gamma)>0,|x|<\frac{1}{k},|y|<\frac{1}{k},\left|\frac{x}{1-k t}\right|<\frac{1}{k}$ and $\left|\frac{y}{1-k t}\right|<\frac{1}{k}$, directly from the properties (36), (39), and (40), we can obtain

$$
\begin{aligned}
& \sum_{n=0}^{\infty} \frac{(\lambda)_{n, k}}{n !}{ }_{2} F_{1, k}\left[\begin{array}{cc}
\lambda+n k, & \alpha \\
\beta & ; x
\end{array}\right]{ }_{2} F_{1, k}\left[\begin{array}{cc}
\lambda+n k, & \gamma \\
\delta & ; y
\end{array}\right] t^{n} \\
= & (1-k t)^{-\frac{\lambda}{k}} \sum_{n=0}^{\infty} \frac{(\lambda)_{n, k}(\alpha)_{n, k}(\gamma)_{n, k}}{(\beta)_{n, k}(\delta)_{n, k} n !}\left(\frac{k^{3} x y t}{(1-k t)^{2}}\right)^{n} \\
& \times{ }_{2} F_{1, k}\left[\begin{array}{c}
\lambda+n k, \alpha+n k \\
\beta+n k
\end{array} \frac{x}{1-k t}\right]{ }_{2} F_{1, k}\left[\begin{array}{c}
\lambda+n k, \\
\delta+n k
\end{array}\right.
\end{aligned}
$$


For the special case $\beta=\delta=\lambda$ in (78), we have

$$
\begin{aligned}
& \sum_{n=0}^{\infty} \frac{(\lambda)_{n, k}}{n !}{ }_{2} F_{1, k}\left[\begin{array}{cc}
\lambda+n k, & \alpha \\
\lambda & ; x
\end{array}\right]{ }_{2} F_{1, k}\left[\begin{array}{cc}
\lambda+n k, & \gamma ; y] t^{n} \\
\lambda &
\end{array}\right] \\
= & (1-k t)^{-\frac{\lambda}{k}} \\
& \times \sum_{n=0}^{\infty} \frac{(\alpha)_{n, k}(\gamma)_{n, k}}{(\lambda)_{n, k} n !}\left(\frac{k^{3} x y t}{(1-k t)^{2}}\right)^{n}\left(1-\frac{k x}{1-k t}\right)^{-\frac{\alpha+n k}{k}}\left(1-\frac{k y}{1-k t}\right)^{-\frac{\gamma+n k}{k}} \\
= & (1-k t)^{\frac{\gamma+\alpha-\lambda}{k}}(1-k t-k x)^{-\frac{\alpha}{k}}(1-k t-k y)^{-\frac{\gamma}{k}} \\
& \times{ }_{2} F_{1, k}\left[\begin{array}{c}
\alpha, \gamma \\
\lambda
\end{array} \frac{k^{3} x y t}{(1-k t-k x)(1-k t-k y)}\right] .
\end{aligned}
$$

\section{Conclusions}

Hypergeometric functions play an important role in many disciplines from different perspectives. Therefore, generalizations of hypergeometric functions have considerable popularity in many fields of science. This work is generally based on the $k$-extension of hypergeometric functions. By making use of the concept of the [26,27], we focus on the generalization of the Appell functions and present some transformation and reduction formulas. Using the theory of Riemann-Liouville $k$-fractional derivative and combining this theory with the Appell functions, we derive some linear and bilinear generating functions.

Author Contributions: Investigation Ö.G.Y., R.A., and F.T.; writing-original draft Ö.G.Y. and R.A.; writing-review and editing F.T. All authors have read and agreed to the published version of the manuscript.

Funding: This research received no external funding.

Conflicts of Interest: The authors declare no conflict of interest.

\section{References}

1. Slater, L.J. Generalized Hypergeometric Functions; Cambridge University Press: Cambridge, UK, 1966.

2. Srivastava, H.; Manocha, H. Treatise on Generating Functions; John Wiley \& Sons, Inc.: New York, NY, USA, 1984.

3. Berends, F.A.; Davydychev, A.I.; Smirnov, V.A. Small-threshold behaviour of two-loop self-energy diagrams: Two-particle thresholds. Nucl. Phys. B 1996, 478, 59-89. [CrossRef]

4. Shpot, M.A. A massive Feynman integral and some reduction relations for Appell functions. J. Math. Phys. 2007, 48, 12. [CrossRef]

5. Włodarczyk, M.; Zawadzki, A. The application of hypergeometric functions to computing fractional order derivatives of sinusoidal functions. Bull. Pol. Acad. Sci. Tech. Sci. 2016, 64, 17. [CrossRef]

6. Chipindirwi, S. Analysis of A Simple Gene Expression Model. M.S. Thesis, University of Zimbabwe, Harare, Zimbabwe, 2012.

7. Abd-Elhameed, W.M. Linearization coefficients of some particular Jacobi polynomials via hypergeometric functions. Adv. Differ. Equ. 2016, 2016, 1-13. [CrossRef]

8. Fröhlich, J. Parameter derivatives of the Jacoby polynomials and the gaussian hypergeometric function. Integral Transform. Spec. Funct. 1994, 2, 253-266. [CrossRef]

9. Lewanowicz, S. The Hypergeometric Functions Approach to the Connection Problem for the Classical Orthogonal Polynomials; University of Wroclaw: Wroclaw, Poland, 2003.

10. Ancarani, L.U.; Del Punta, J.A.; Gasaneo, G. Derivatives of Horn hypergeometric functions with respect to their parameters. J. Math. Phys. 2017, 58, 127-165. [CrossRef]

11. Dragovic, V. The Appell hypergeometric functions and classical separable mechanical systems. J. Phys. Math. Gen. 2002, 35, 84-97. [CrossRef] 
12. Colavecchia, F.D.; Gasaneo, G.; Garibotti, C.R. Hypergeometric integrals arising in atomic collisions physics. J. Math. Phys. 1997, 38, 567-583. [CrossRef]

13. Diaz, R.; Pariguan, E. On hypergeometric functions and pochhammer k-symbol. Divulg. Mat. 2007, 15, 179-192.

14. Kokologiannaki, C.G. Properties and inequalities of generalized $k$-gamma, beta and zeta functions. Int. Contemp. Math. Sci. 2010, 5, 653-660.

15. Krasniqi, V. A limit for the k-gamma and k-beta function. Int. Math. Forum. 2010, 5, 1613-1617.

16. Mubeen, S.; Habibullah, G. An integral representation of some k-hypergeometric functions. Int. Math. Forum. 2012, 7, 203-207.

17. Mubeen, S. k-analogue of Kummer's first formula. J. Inequalities Spec. Funct. 2012, 3, 41-44.

18. Korkmaz-Duzgun, D.; Erkus-Duman, E. Generating functions for k-hypergeometric functions. Int. J. Appl. Phys. Math. 2019, 9, 119-126.

19. Chinra, S.; Kamalappan, V.; Rakha, M.A.; Rathie, A.K. On several new contiguous function relations for k-hypergeometric function with two parameters. Commun. Korean Math. Soc. 2017, 32, 637-651.

20. Li, S.; Dong, Y. $k$-hypergeometric series solutions to one type of non-homogeneous $k$-hypergeometric equations. Symmetry 2019, 11, 262. [CrossRef]

21. Nisar, K.S.; Qi, F.; Rahman, G.; Mubeen, S.; Arshad, M. Some inequalities involving the extended gamma function and the Kummer confluent hypergeometric $k$-function. J. Inequalities Appl. 2018, 2018, 1-12. [CrossRef]

22. Mubeen, S.; Rehman, A. A note on k-gamma function and pochhammer k-symbol, J. Informatics Math. Sci. 2014, 6, 93-107.

23. Mubeen, S.; Habibullah, G. $k$-fractional integrals and application. Int. J. Contemp. Math. Sci. 2012, 7, 89-94.

24. Romero, L.G.; Luque, L.L.; Dorrego, G.A.; Cerutti, R.A. On the $k$-Riemann-Liouville fractional derivative. Int. Contemp. Math. Sci. 2013, 8, 41-51. [CrossRef]

25. Rahman, G.; Nisar Mubeen, S.; Sooppy, K. On generalized k-fractional derivative operator. AIMS Math. 2020, 5, 1936-1945. [CrossRef]

26. Mubeen, S.; Iqbal, S.; Rahman, G. Contiguous function relations and an integral representation for Appell k-series. Int. Math. Res. 2015, 4, 53-63. [CrossRef]

27. Kıymaz, İ. O.; Çetinkaya, A.; Agarwal, P. A study on the k-generalizations of some known functions and fractional operators. J. Inequal. Spec. Funct. 2017, 8, 31-41.

28. Özarslan, M.A.; Ustaoglu, C. Incomplete Caputo fractional derivative operators. Adv. Differ. Equ. 2018, 2018, 209. [CrossRef]

29. Özarslan, M.A.; Ustaoglu, C. Some Incomplete Hypergeometric Functions and Incomplete Riemann-Liouville Fractional Integral Operators. Mathematics 2019, 7, 483. [CrossRef]

30. Srivastava, H.M.; Parmar, R.K.; Chopra, P. A class of extended fractional derivative operators and associated generating relations involving hypergeometric functions. Axioms 2012, 1, 238-258. [CrossRef]

31. Choi, J.; Agarwal, P.; Jain, S. Certain fractional integral operators and extended generalized Gauss hypergeometric functions. Kyungpook Math. J. 2015, 55, 695-703. [CrossRef]

32. Özarslan, M.A.; Kürt, C. Nonhomogeneous initial and boundary value problem for the caputo-type fractional wave equation, Adv. Differ. Equ. 2019, 2019, 199. [CrossRef]

33. Fernandez, A.; Kürt, C.; Özarslan, M.A. A naturally emerging bivariate Mittag-Leffler function and associated fractional-calculus operators. Comput. Appl. Math. 2020, 39, 1-27. [CrossRef]

34. Azam, M.; Farid, G.; Rehman, M. Study of generalized type $k$-fractional derivatives. Adv. Differ. Equ. 2017, 2017, 249. [CrossRef]

(C) 2020 by the authors. Licensee MDPI, Basel, Switzerland. This article is an open access article distributed under the terms and conditions of the Creative Commons Attribution (CC BY) license (http:/ / creativecommons.org/licenses/by/4.0/). 